\title{
Hall Effects on Unsteady Rotating MHD Flow Through Porous Channel with Variable Pressure Gradient
}

\author{
S. Das \\ Department of Mathematics \\ University of Gour Banga \\ Malda 732 103, India
}

\author{
H. K. Mandal \\ Department of \\ Applied Mathematics \\ Vidyasagar University \\ Midnapore 721 102, India
}

\author{
R. N. Jana \\ Department of \\ Applied Mathematics \\ Vidyasagar University \\ Midnapore 721 102, India
}

\begin{abstract}
Hall effects on an unsteady MHD flow of a viscous incompressible electrically conducting fluid in a horizontal porous channel with variable pressure gradient in a rotating system have been studied. We have considered three different cases (i) impulsive change of pressure gradient (ii) cosine oscillations of pressure gradient and (iii) sine oscillations of pressure gradient. The governing equations are solved analytically using the Laplace transform technique. It is found that interplay of Coriolis force and hydromagnetic force in the presence of pressure gradient and Hall currents plays an important role in characterizing the flow behavior. Effects of the parameters of these forces on the velocity distributions and shear stresses have been depicted graphically and discussed. It is found that the primary velocity increases with an increase in Hall parameter for the impulsive change, cosine and sine oscillations of the pressure gradient. The secondary velocity increases for the impulsive change and cosine oscillations of the pressure gradient while it decreases for sine oscillations of the pressure gradient with an increase in Hall parameter. Further, the shear stress due to the primary flow at the lower wall reduces for both the impulsive change and cosine oscillations of the pressure gradient whereas it increases for sine oscillations of the pressure gradient with an increase in Hall parameter.
\end{abstract}

Keywords: MHD flow, Hall currents, rotation parameter, Reynolds number, frequency parameter, pressure gradient, impulsive change, cosine oscillations and sine oscillations.

\section{INTRODUCTION}

The rotating flow of an electrically conducting fluid in the presence of a magnetic field is encountered in cosmical and geophysical fluid dynamics. It can provide an explanation for the observed maintenance and secular variation of the geomagnetic field (Hide and Roberts [1]). It is also important in the solar physics involved in the sunspot development, the solar cycle and the structure of rotating magnetic stars (Dieke [2]). The effect of the Coriolis force due to the earth's rotation is found to be significant as compared to the inertial and viscous forces in the equations of motion. The Coriolis and the electromagnetic forces are of comparable magnitude. The Coriolis force exerts a strong influence on the hydromagnetic flow in the earth's liquid core which plays an important role in the mean geomagnetic field. The magnetohydrodynamic rotating flow of electrically conducting viscous incompressible fluids has gained considerable attention because of its numerous applications in physics and engineering. In geophysics, it is applied to measure and study the positions and velocities with respect to a fixed frame of reference on the surface of the earth, which rotate with respect to an inertial frame in the presence of its magnetic field. The subject of geophysical dynamics now-a-days has become an important branch of fluid dynamics due to the increasing interest to study environment. In astrophysics, it is applied to study the stellar and solar structure, inter planetary and inter stellar matter, solar storms etc. In engineering, it finds its application in MHD generators, ion propulsion, MHD bearings, MHD pumps, MHD boundary layer control of re-entry vehicles etc. In some astronomical and geophysical problems and in many engineering applications the study of Coriolis force interaction with electromagnetic force is important. Hall effect is also important when the fluid is an ionized gas with low density or the applied magnetic field is very strong. Because the electrical conductivity of the fluid will then be a tensor and a current (Hall current) is induced which is likely to be important in many engineering situations (Sutton and Sherman [3]). The Hall effects on the flow of ionized gas between parallel plates under transverse magnetic field have been studied by Sato [4]. Nanda and Mohanty [5] studied the hydromagnetic rotating channel flows. Datta and Jana [6] have discussed Hall effects on unsteady Couette flow. Hall effects on hydromagnetic convective flow through a channel with conducting walls have been studied by Datta and Jana [7]. Mandal et al. [8] have analyzed the combined effects of Coriolis force and Hall current on steady MHD Couette flow and heat transfer. Effects of Hall current on MHD Couette flow between thick arbitrarily conducting plates in a rotating system have been studied by Mandal and Mandal [9]. Ghosh [10] has studied the unsteady hydromagnetic flow in a rotating channel with oscillating pressure gradient. Effects of Hall currents and Coriolis force on Hartmann flow under general wall conditions have been analyzed by Nagy and Demandy [11]. Kanch and Jana [12] have studied the Hall effect on unsteady Couette flow under boundary layer approximations. Effects of Hall current on MHD Couette flow in a rotating system with arbitrary magnetic field have been investigated by Ghosh [13]. Hall effects on MHD plasma Couette flow in a rotating environment have been studied by Ghosh and Pop [14]. Ghosh [15] has investigated the Hall effects on an MHD Couette flow in a rotating system with arbitrary magnetic field. Hall effects on the hydromagnetic convective flow through a rotating channel under general wall conditions have been examined by Guria and Jana [16]. Attia [17] have studied the ion-slip effects on unsteady Couette flow with heat transfer under exponential decaying pressure gradient. Seth et al. [18] have studied the Hall effects on oscillatory hydromagnetic Couette flow in a rotating system. Hall effects on an MHD flow in a rotating channel partially filled with a porous medium have been examined by Chauhan and Rastogi [19] and Chauhan and Agrawal [20]. The combined effects of Hall current and ion-slip current on unsteady MHD Couette flow in a rotating system have been investigated by Jha and Apere [21]. Guchhait et al. [22] have studied the combined effects of Hall currents and rotation on unsteady Couette flow in a porous 
channel. Hall effects on MHD Couette flow in rotating system have been studied by Das et al. [23]. Ghara et al. [24] have studied the effects of Hall currents and ion-slip on unsteady MHD Couette flow. Effects of Hall currents and rotation on unsteady MHD Couette flow in the presence of an inclined magnetic field have been studied by Seth et al. [25]. Chauhan and Agrawal [26] have studied the Hall effects on an MHD Couette flow in a channel partially filled with a porous medium in a rotating system. Sarkar et al. [27] have examined the combined effects of Hall currents and rotation on steady hydromagnetic Couette flow. The numerical solutions of peristaltic flow of a newtonian fluid under the effects of magnetic field and heat transfer in a porous concentric tubes have been studied by Nadeem et al.[28]. Nadeem and Akbar [29] have discussed the influence of heat transfer and variable viscosity in vertical porous annulus with peristalsis. Nadeem et al. [30] have studied the influence of heat and mass transfer on Newtonian bio-magnetic fluid of blood flow through a tapered porous arteries with a stenosis. The simulation of variable viscosity and Jeffrey fluid model for blood flow through a tapered artery with a stenosis has been presented by Akbar and Nadeem [31]. [32] Akbar and Nadeem have presented the analytical and numerical analysis of Vogel's model of viscosity on the peristaltic flow of Jeffrey fluid.

The aim of the present paper is to study the Hall effects on the unsteady MHD flow of a viscous incompressible electrically conducting fluid in a horizontal porous channel in a rotating system. It is found that the primary velocity $u_{1}$ increases with an increase in Hall parameter $m$ for the impulsive change, cosine and sine oscillations of the pressure gradient. The secondary velocity $w_{1}$ increases for the impulsive change and cosine oscillations while it decreases for sine oscillations of the pressure gradient with an increase in Hall parameter $m$. The primary and secondary velocities decrease with an increase in rotation parameter $K^{2}$ for the impulsive change, cosine and sine oscillations of the pressure gradient. Further, the shear stress $\tau_{x}$

due to the primary flow at the lower wall reduces for both the impulsive change and cosine oscillations of the pressure whereas it increases for sine oscillations of the pressure gradient with an increase in Hall parameter $m$. The shear stress $\tau_{y}$ due to the secondary flow at the lower wall increases with an increase in Hall parameter $m$ for the impulsive change, cosine and sine oscillations of the pressure gradient.

\section{MATHEMATICAL FORMULATION AND ITS SOLUTION}

Consider the unsteady MHD flow of a viscous incompressible electrically conducting fluid between two infinitely long horizontal parallel walls separated by a distance $h$. Choose a Cartesian co-ordinates system with the $x$-axis along the channel wall at $y=0$, the $y$-axis perpendicular to the channel walls and $z$-axis is normal to the $x y$-plane (See Fig.1). A uniform transverse magnetic field $H_{0}$ is applied perpendicular to the channel walls. Since the channel walls are infinite in extent and the flow is unsteady, the physical variables are the function of $y$ and $t$ only.

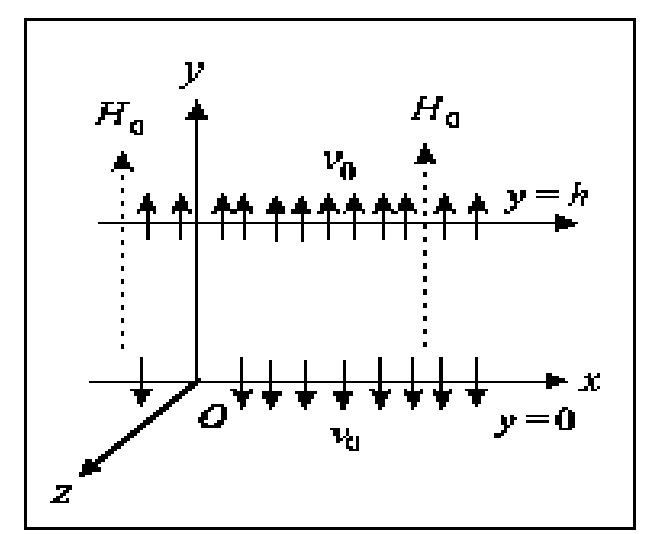

Fig.1 : Geometry of the problem

The governing equations of motion along $x$ and $z$-directions in a rotating frame of reference are

$$
\begin{aligned}
& \frac{\partial u}{\partial t}-v_{0} \frac{\partial u}{\partial y}+2 \Omega w=-\frac{1}{\rho} \frac{\partial p}{\partial x}+v \frac{\partial^{2} u}{\partial y^{2}}-\frac{\sigma \mu_{e} H_{0}}{\rho} j_{z}, \\
& \frac{\partial w}{\partial t}-v_{0} \frac{\partial w}{\partial y}-2 \Omega u=v \frac{\partial^{2} u}{\partial y^{2}}-\frac{\sigma \mu_{e} H_{0}}{\rho} j_{x}
\end{aligned}
$$

where $\rho$ is the fluid density, $v$ the kinematic viscosity, $u$ and $w$ are fluid velocity components and $p$ the fluid pressure including centrifugal force.

The initial and boundary conditions are

$$
\begin{aligned}
& t \leq 0: u=v=w=0 \text { for } 0 \leq y \leq h, \\
& t>0: u=0, w=0, v=v_{0} \text { at } y=0, \\
& t>0: u=w=0, v=v_{0} \text { at } y=h .
\end{aligned}
$$

The generalized Ohm's law comes essentially from the momentum equation of motion for the electron fluid. Its derivation can be found in some plasma physics books (like Goldston). It can be written, on taking Hall currents into account and neglecting ionslip and thermo-electric effect, as (see Cowling [33])

$$
\vec{j}+\frac{\omega_{e} \tau_{e}}{H_{0}}(\vec{j} \times \vec{H})=\sigma\left(\vec{E}+\mu_{e} \vec{q} \times \vec{H}\right),
$$

where $\vec{j}$ is the current density vector, $\vec{H}$ the magnetic field vector, $\vec{E}$ the electric field vector, $\omega_{e}$ the cyclotron frequency, $\sigma$ the electrical conductivity of the fluid, $\tau_{e}$ the collision time of electron and $\mu_{e}$ the magnetic permeability. The right hand side is the electric field in the moving frame. The first term on the left hand side comes from the electron drag on the ions. The second term is the Hall term and has to do with the idea that electrons and ions can decouple and move separately.

It is assumed that the magnetic Reynolds number is small, so that the induced magnetic field is negligible in comparison to the applied magnetic field. The electron atom collision frequency is assumed to be relatively high as compared to the collision frequency of ions and so electron pressure gradient term is also neglected but Hall effect is assumed to exist. This assumption is justified since the magnetic Reynolds number is generally very small for partially ionized gases. The solenoidal relation $\nabla \cdot \vec{H}=0$ for the magnetic field gives $H_{y}=H_{0}=$ constant everywhere in the fluid where $\vec{H} \equiv\left(0, H_{0}, 0\right)$. Further, if 
$\left(j_{x}, j_{y}, j_{z}\right)$ be the components of the current density $\vec{j}$, then the equation of the conservation of the current density $\nabla \cdot \vec{j}=0$ gives $j_{y}=$ constant . This constant is zero since $j_{y}=0$ at the plates which are electrically non-conducting. Thus $j_{y}=0$ everywhere in the flow. Since the induced magnetic field is neglected, Maxwell's equation $\nabla \times \vec{E}=-\frac{\partial \vec{H}}{\partial t}$ becomes $\nabla \times \vec{E}=0$ which gives $\frac{\partial E_{x}}{\partial y}=0$ and $\frac{\partial E_{y}}{\partial y}=0$. This implies that $E_{x}=$ constant and $E_{z}=$ constant everywhere in the flow. We choose this constants equal to zero, i.e. $E_{x}=E_{z}=0$.

In view of the above assumption, the equation (4) gives

$$
\begin{aligned}
& j_{x}-m j_{z}=-\sigma \mu_{e} H_{0} w, \\
& j_{z}+m j_{x}=-\sigma \mu_{e} H_{0} u,
\end{aligned}
$$

where $m=\omega_{e} \tau_{e}$ is the Hall parameter.

Solving (5) and (6) for $j_{x}$ and $j_{z}$, we have

$$
\begin{aligned}
& j_{x}=-\frac{\sigma \mu_{e} H_{0}}{1+m^{2}}(w-m u), \\
& j_{z}=\frac{\sigma \mu_{e} H_{0}}{1+m^{2}}(u+m w) .
\end{aligned}
$$

On the use of (7) and (8), the momentum equations (1) and (2) along $x$ - and $z$-directions become

$$
\begin{aligned}
& \frac{\partial u}{\partial t}-v_{0} \frac{\partial u}{\partial y}+2 \Omega w=-\frac{1}{\rho} \frac{\partial p}{\partial x}+v \frac{\partial^{2} u}{\partial y^{2}}-\frac{\sigma \mu_{e}^{2} H_{0}^{2}}{\rho\left(1+m^{2}\right)}(u+m w), \\
& \frac{\partial w}{\partial t}-v_{0} \frac{\partial w}{\partial y}-2 \Omega u=v \frac{\partial^{2} u}{\partial y^{2}}-\frac{\sigma \mu_{e}^{2} H_{0}^{2}}{\rho\left(1+m^{2}\right)}(w-m u),
\end{aligned}
$$

Introducing non-dimensional variables

$$
\left(u_{1}, w_{1}\right)=\frac{(u, w) h}{v}, \eta=\frac{y}{h}, \tau=\frac{v t}{h^{2}},
$$

equations (9) and (10) become

$$
\begin{aligned}
& \frac{\partial u_{1}}{\partial \tau}-\operatorname{Re} \frac{\partial u_{1}}{\partial \eta}+2 K^{2} w_{1}=f(\tau)+\frac{\partial^{2} u_{1}}{\partial \eta^{2}}-\frac{M^{2}}{1+m^{2}}\left(u_{1}+m w_{1}\right), \\
& \frac{\partial w_{1}}{\partial \tau}-\operatorname{Re} \frac{\partial w_{1}}{\partial \eta}-2 K^{2} u_{1}=\frac{\partial^{2} w_{1}}{\partial \eta^{2}}-\frac{M^{2}}{1+m^{2}}\left(w_{1}-m u_{1}\right),
\end{aligned}
$$

where $M^{2}=\frac{\sigma \mu_{e}{ }^{2} B_{0}{ }^{2} h^{2}}{\rho v}$ is the magnetic parameter, $K^{2}=\frac{\Omega^{2} h^{2}}{v}$ the rotation parameter, $R e=\frac{v_{0} h}{v}$ Reynolds number and $f(\tau)=-\frac{\partial p^{*}}{\partial x}$ the non-dimensional pressure gradient.

The initial and boundary conditions (3) become

$$
\begin{aligned}
& \tau \leq 0: u_{1}=w_{1}=0 \text { for } 0 \leq \eta \leq 1, \\
& \tau>0: u_{1}=0, w_{1}=0 \text { at } \eta=0, \\
& \tau>0: u_{1}=w_{1}=0 \text { at } \eta=1 .
\end{aligned}
$$

Combining equations (12) and (13), we get

$$
\frac{\partial F}{\partial \tau}-\operatorname{Re} \frac{\partial F}{\partial \eta}-2 i K^{2} F=f(\tau)+\frac{\partial^{2} F}{\partial \eta^{2}}-\frac{(1-i m) M^{2}}{1+m^{2}} F,
$$

where

$$
F=u_{1}+i w_{1} \text { and } i=\sqrt{-1} .
$$

The boundary conditions for $F(\eta, \tau)$ are

$$
\begin{aligned}
& \tau \leq 0: F=0 \text { for } 0 \leq \eta \leq 1, \\
& \tau>0: F=0 \text { at } \eta=0, \\
& \tau>0: F=0 \text { at } \eta=1 .
\end{aligned}
$$

Taking the Laplace transform of the equation (15), we have

$$
\frac{d^{2} \bar{F}}{d \eta^{2}}+\operatorname{Re} \frac{d \bar{F}}{\partial \eta}-\left[\frac{(1-i m) M^{2}}{1+m^{2}}-2 i K^{2}+s\right] \bar{F}=-\bar{f}(s),
$$

where $\bar{f}(s)$ is the Laplace transform of $f(\tau)$.

The initial and boundary conditions for $\bar{F}(\eta, s)$ are

$$
\bar{F}(0, s)=0 \text { and } \bar{F}(1, s)=0 .
$$

Solutions of the equation (18) subject to the boundary conditions (19) are given by

$$
\begin{gathered}
\bar{F}(\eta, s)=\frac{\bar{f}(s)}{d+s}\left[1-e^{-\frac{1}{2} \operatorname{Re\eta } \sinh \sqrt{r+s}(1-\eta)}\right. \\
\sinh \sqrt{r+s} \\
\left.-e^{\frac{1}{2} \operatorname{Re}(1-\eta)} \frac{\sinh \sqrt{r+s} \eta}{\sinh \sqrt{r+s}}\right],
\end{gathered}
$$

where

$$
d=\frac{M^{2}(1-i m)}{1+m^{2}}-2 i K^{2} \text { and } r=\frac{R e^{2}}{4}+d .
$$

We assume

$$
f(t)=P_{0}+P_{1} e^{i \omega^{*} t}+P_{2} e^{-i \omega^{*} t},
$$

where $\omega^{*}$ is the frequency of oscillations and $P_{0}, P_{1}$ and $P_{2}$ are constants.

The inverse Laplace transforms of the equation (20) gives the solution for the velocity field as

$$
\begin{aligned}
& F(\eta, \tau)=\frac{P_{0}}{d}\left[1-e^{-\frac{1}{2} R e \eta} \frac{\sinh (\alpha-i \beta)(1-\eta)}{\sinh (\alpha-i \beta)}\right. \\
& \left.-e^{\frac{1}{2} \operatorname{Re}(1-\eta)} \frac{\sinh (\alpha-i \beta) \eta}{\sinh (\alpha-i \beta)}\right] \\
& +\frac{P_{1}}{d+i n}\left[1-e^{-\frac{1}{2} \operatorname{Re} \eta} \frac{\sinh \left(\alpha_{1} \mp i \beta_{1}\right)(1-\eta)}{\sinh \left(\alpha_{1} \mp i \beta_{1}\right)}\right. \\
& \left.-e^{\frac{1}{2} \operatorname{Re}(1-\eta)} \frac{\sinh \left(\alpha_{1} \mp i \beta_{1}\right) \eta}{\sinh \left(\alpha_{1} \mp i \beta_{1}\right)}\right] e^{i \omega \tau} \\
& +\frac{P_{2}}{d-i n}\left[1-e^{-\frac{1}{2} \operatorname{Re} \eta} \frac{\sinh \left(\alpha_{2}-i \beta_{2}\right)(1-\eta)}{\sinh \left(\alpha_{2}-i \beta_{2}\right)}\right. \\
& \left.-e^{\frac{1}{2} \operatorname{Re}(1-\eta)} \frac{\sinh \left(\alpha_{2}-i \beta_{2}\right) \eta}{\sinh \left(\alpha_{2}-i \beta_{2}\right)}\right] e^{-i \omega \tau} \\
& -\left(\frac{P_{0}}{d}+\frac{P_{1}}{d+i n}+\frac{P_{2}}{d-i n}\right)
\end{aligned}
$$

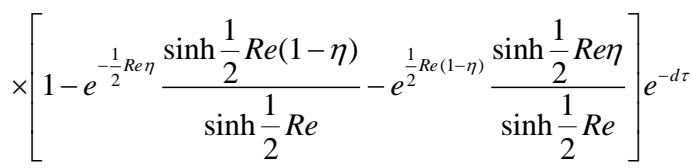

$$
\begin{aligned}
& +2 \sum_{n=1}^{\infty} n \pi\left(\frac{P_{0}}{s_{1}}+\frac{P_{1}}{s_{1}-i \omega}+\frac{P_{2}}{s_{1}+i \omega}\right)
\end{aligned}
$$




$$
\times\left(\frac{e^{s_{1} \tau}}{d+s_{1}}\right)\left[(-1)^{n} e^{\frac{1}{2} R e(1-\eta)}-e^{-\frac{1}{2} R e \eta}\right] \sin n \pi \eta,
$$

where

$$
\begin{aligned}
s_{1} & =-\left[n^{2} \pi^{2}+\frac{R e^{2}}{4}+\frac{(1-i m) M^{2}}{1+m^{2}}-2 i K^{2}\right] \\
\alpha, \beta & =\frac{1}{\sqrt{2}}\left[\left\{\left(\frac{R e^{2}}{4}+\frac{M^{2}}{1+m^{2}}\right)^{2}+\left(2 K^{2}+\frac{m M^{2}}{1+m^{2}}\right)^{2}\right\}^{1 / 2}\right. \\
& \left. \pm\left(\frac{R e^{2}}{4}+\frac{M^{2}}{1+m^{2}}\right)\right]^{1 / 2}, \\
\alpha_{1}, \beta_{1} & =\frac{1}{\sqrt{2}}\left[\left\{\left(\frac{R e^{2}}{4}+\frac{M^{2}}{1+m^{2}}\right)^{2}+\left(2 K^{2}+\frac{m M^{2}}{1+m^{2}}-\omega\right)^{2}\right\}^{1 / 2}\right. \\
& \left. \pm\left(\frac{R^{2}}{4}+\frac{M^{2}}{1+m^{2}}\right)\right]^{1 / 2}, \\
\alpha_{2}, \beta_{2} & =\frac{1}{\sqrt{2}}\left[\left\{\left(\frac{R e^{2}}{4}+\frac{M^{2}}{1+m^{2}}\right)^{2}+\left(2 K^{2}+\frac{m M^{2}}{1+m^{2}}+\omega\right)^{2}\right\}^{1 / 2}\right. \\
& \left. \pm\left(\frac{R e^{2}}{4}+\frac{M^{2}}{1+m^{2}}\right)\right]^{1 / 2} \cdot
\end{aligned}
$$

In the equation (23), the upper sign is for $2 K^{2}+\frac{m M^{2}}{1+m^{2}}>\omega$ and the lower sign is for $2 K^{2}+\frac{m M^{2}}{1+m^{2}}<\omega$. The equation (23) describes the fluid velocities in the general case. Now, we shall considered the following cases.

(i) Velocity distribution for impulsive pressure gradient: In this case $P_{1}=P_{2}=0$. Then the equation (24) becomes

$$
\begin{aligned}
& F(\eta, \tau)=\frac{P_{0}}{d}\left[1-e^{-\frac{1}{2} \operatorname{Re} \eta} \frac{\sinh (\alpha-i \beta)(1-\eta)}{\sinh (\alpha-i \beta)}\right. \\
& \left.-e^{\frac{1}{2} \operatorname{Re}(1-\eta)} \frac{\sinh (\alpha-i \beta) \eta}{\sinh (\alpha-i \beta)}\right] \\
& -\frac{P_{0}}{d}\left[1-e^{-\frac{1}{2} R e \eta} \frac{\sinh \frac{1}{2} \operatorname{Re}(1-\eta)}{\sinh \frac{1}{2} R}-e^{\frac{1}{2} R e(1-\eta)} \frac{\sinh \frac{1}{2} R \eta}{\sinh \frac{1}{2} R e}\right] e^{-d \tau} \\
& +2 P_{0} \pi \sum_{n=1}^{\infty} \frac{n}{s_{1}}\left(\frac{e^{s_{1} \tau}}{d+s_{1}}\right)\left[(-1)^{n} e^{\frac{1}{2} R e(1-\eta)}-e^{-\frac{1}{2} R e \eta}\right] \sin n \pi \eta,
\end{aligned}
$$

where $\alpha, \beta$ and $s_{1}$ are given by (24).

(ii) Velocity distribution for cosine oscillations of pressure gradient:

In this case $P_{0}=0$ and $P_{1}=P_{2}=\frac{P}{2}$. Then the equation (23) becomes

$$
F(\eta, \tau)=\frac{P}{2}\left[\frac { e ^ { i \omega \tau } } { d + i \omega } \left\{1-e^{-\frac{1}{2} R e \eta} \frac{\sinh \left(\alpha_{1} \mp i \beta_{1}\right)(1-\eta)}{\sinh \left(\alpha_{1} \mp i \beta_{1}\right)}\right.\right.
$$

$$
\begin{aligned}
& \left.-e^{\frac{1}{2} R e(1-\eta)} \frac{\sinh \left(\alpha_{1} \mp i \beta_{1}\right) \eta}{\sinh \left(\alpha_{1} \mp i \beta_{1}\right)}\right\} \\
& +\frac{e^{-i \omega \tau}}{d-i \omega}\left\{1-e^{-\frac{1}{2} \operatorname{Re\eta } \frac{\sinh \left(\alpha_{2}-i \beta_{2}\right)(1-\eta)}{\sinh \left(\alpha_{2}-i \beta_{2}\right)}}\right. \\
& \left.\left.-e^{\frac{1}{2} R e(1-\eta)} \frac{\sinh \left(\alpha_{2}-i \beta_{2}\right) \eta}{\sinh \left(\alpha_{2}-i \beta_{2}\right)}\right\}\right] \\
& -\frac{P}{2}\left(\frac{1}{d+i \omega}+\frac{1}{d-i \omega}\right) \\
& \times\left[1-e^{-\frac{1}{2} \operatorname{Re\eta }} \frac{\sinh \frac{1}{2} \operatorname{Re}(1-\eta)}{\sinh \frac{1}{2} \operatorname{Re}}-e^{\frac{1}{2} R e(1-\eta)} \frac{\sinh \frac{1}{2} \operatorname{Re\eta }}{\left.\sinh \frac{1}{2} \operatorname{Re}\right] e^{-d \tau}}\right. \\
& +\pi P \sum_{n=1}^{\infty} n\left(\frac{1}{s_{1}-i \omega}+\frac{1}{s_{1}+i \omega}\right) \\
& \times\left(\frac{e^{s_{1} \tau}}{d+s_{1}}\right)\left[(-1)^{n} e^{\frac{1}{2} R(1-\eta)}-e^{-\frac{1}{2} R e \eta}\right] \sin n \pi \eta
\end{aligned}
$$

where $\alpha_{1}, \beta_{1}, \alpha_{2}, \beta_{2}$ and $s_{1}$ are given by (24).

(iii) Velocity distribution for sine oscillations of pressure gradient:

In this case $P_{0}=0$ and $P_{1}=P_{2}=\frac{P}{2 i}$. Then the equation (23) becomes

$$
\begin{aligned}
& F(\eta, \tau)=\frac{P}{2 i}\left[\left\{1-e^{-\frac{1}{2} R e \eta} \frac{\sinh \left(\alpha_{1} \mp i \beta_{1}\right)(1-\eta)}{\sinh \left(\alpha_{1} \mp i \beta_{1}\right)}\right.\right. \\
& \left.-e^{\frac{1}{2} \operatorname{Re}(1-\eta)} \frac{\sinh \left(\alpha_{1} \mp i \beta_{1}\right) \eta}{\sinh \left(\alpha_{1} \mp i \beta_{1}\right)}\right\} \\
& +\frac{e^{-i \omega \tau}}{d-i \omega}\left\{1-e^{-\frac{1}{2} \operatorname{Re} \eta} \frac{\sinh \left(\alpha_{2}-i \beta_{2}\right)(1-\eta)}{\sinh \left(\alpha_{2}-i \beta_{2}\right)}\right. \\
& \left.\left.-e^{\frac{1}{2} \operatorname{Re}(1-\eta)} \frac{\sinh \left(\alpha_{2}-i \beta_{2}\right) \eta}{\sinh \left(\alpha_{2}-i \beta_{2}\right)}\right\}\right] \\
& -\frac{P}{2 i}\left(\frac{1}{d+i \omega}+\frac{1}{d-i \omega}\right) \\
& \times\left[1-e^{-\frac{1}{2} \operatorname{Re\eta }} \frac{\sinh \frac{1}{2} \operatorname{Re}(1-\eta)}{\sinh \frac{1}{2} \operatorname{Re}}-e^{\frac{1}{2} \operatorname{Re}(1-\eta)} \frac{\sinh \frac{1}{2} \operatorname{Re} \eta}{\sinh \frac{1}{2} \operatorname{Re}}\right] e^{-d \tau} \\
& -\pi P i \sum_{n=1}^{\infty} n\left(\frac{1}{s_{1}-i \omega}+\frac{1}{s_{1}+i \omega}\right) \\
& \times\left(\frac{e^{s_{1} \tau}}{d+s_{1}}\right)\left[(-1)^{n} e^{\frac{1}{2} R e(1-\eta)}-e^{-\frac{1}{2} R e \eta}\right] \sin n \pi \eta,
\end{aligned}
$$

where $\alpha_{1}, \beta_{1}, \alpha_{2}, \beta_{2}$ and $s_{1}$ are given by (24).

\section{RESULTS AND DISCUSSION}

We have presented the non-dimensional velocity components for several values of magnetic parameter $M^{2}$, Hall parameter $m$, 
rotation parameter $K^{2}$, Reynolds number $\mathrm{Re}$, frequency parameter $\omega$ and phase angle $\omega \tau$ against $\eta$ in Figs.2-13. It is seen from Fig. 2 that the primary velocity $u_{1}$ decreases with an increase in magnetic parameter $M^{2}$ for the impulsive change, cosine and sine oscillations of the pressure gradient. Fig. 3 shows that the secondary velocity $w_{1}$ decreases for the impulsive change and cosine oscillations of the pressure gradient while it increases for sine oscillations of the pressure gradient with an increase in magnetic parameter $M^{2}$. As expected due to the fact that the application of transverse magnetic field results to a resistive type force (called Lorentz force) similar to drag force and upon increasing the values of magnetic parameter, the drag force increases which leads to the deceleration of the flow. It is seen from Fig. 4 that the primary velocity $u_{1}$ increases with an increase in Hall parameter $m$ for the impulsive change, cosine and sine oscillations of the pressure gradient. Hence, we conclude that an increase in Hall parameter reduces the Lorentz force in $x$ direction and motion of the fluid particles is reinforced in that direction. Fig.5 shows that the secondary velocity $w_{1}$ increases for the impulsive change and cosine oscillations of the pressure gradient while it decreases for sine oscillations of the pressure gradient with an increase in Hall parameter $m$. As reported in numerous MHD studies, this velocity component is a result of the Hall effect. It is seen from Figs. 6 and 7 that the primary velocity $u_{1}$ and the secondary velocity $w_{1}$ decreases with an increase in rotation parameter $K^{2}$ for the impulsive change, cosine and sine oscillations of the pressure gradient. The rotation parameter defines the relative magnitude of the Coriolis force and the viscous force in the regime; therefore it is clear that high magnitude Coriolis forces are counter-productive for the primary flow. Fig. 8 shows that the primary velocity $u_{1}$ decreases with an increase in Reynolds number $\operatorname{Re}$ for the impulsive change, cosine and sine oscillations of the pressure gradient. It is seen from Fig.9 that the secondary velocity $w_{1}$ increases for the impulsive change and cosine oscillations of the pressure gradient while it decreases for sine oscillations of the pressure gradient with an increase in Reynolds number Re. Fig.10 shows that the primary velocity $u_{1}$ increases with an increase in frequency parameter $\omega$ for the impulsive change, cosine and sine oscillations of the pressure gradient. It is seen from Fig. 11 that the secondary velocity $w_{1}$ increases for the impulsive change and cosine oscillations of the pressure gradient while it decreases for sine oscillations of the pressure gradient with an increase in frequency parameter $\omega$. Fig. 12 shows that the primary velocity $u_{1}$ decreases with an increase in phase angle $\omega \tau$ for both cosine and sine oscillations of the pressure gradient. It is seen from Fig.13 that the secondary velocity $w_{1}$ decreases for cosine oscillations of the pressure gradient while it increases for sine oscillations of the pressure gradient with an increase in phase angle $\omega \tau$. It is noted that the velocities for cosine oscillations of the pressure gradient are always greater than sine oscillations of the pressure gradient.

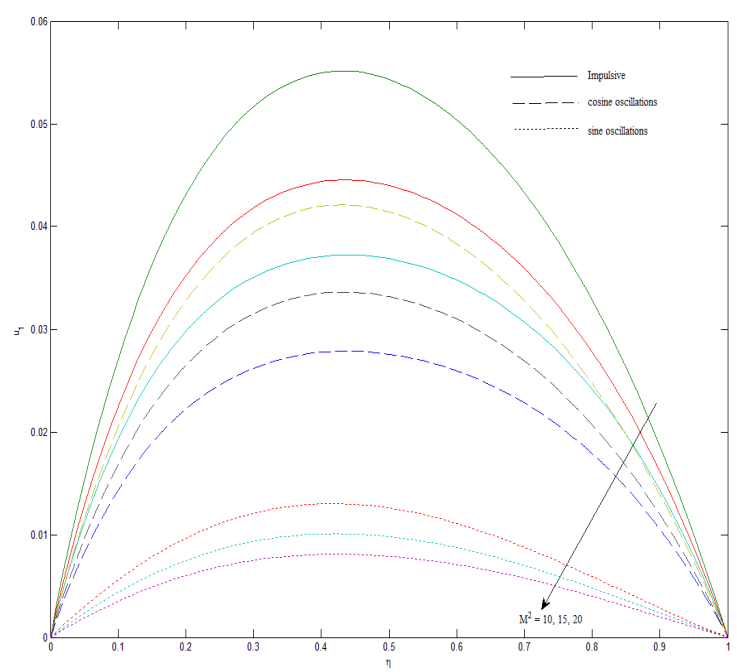

Fig.2: Primary velocity $u_{1}$ for different $M^{2}$ when $R e=2$, $m=0.2, K^{2}=2, \omega=2$ and $\omega \tau=\frac{\pi}{4}$

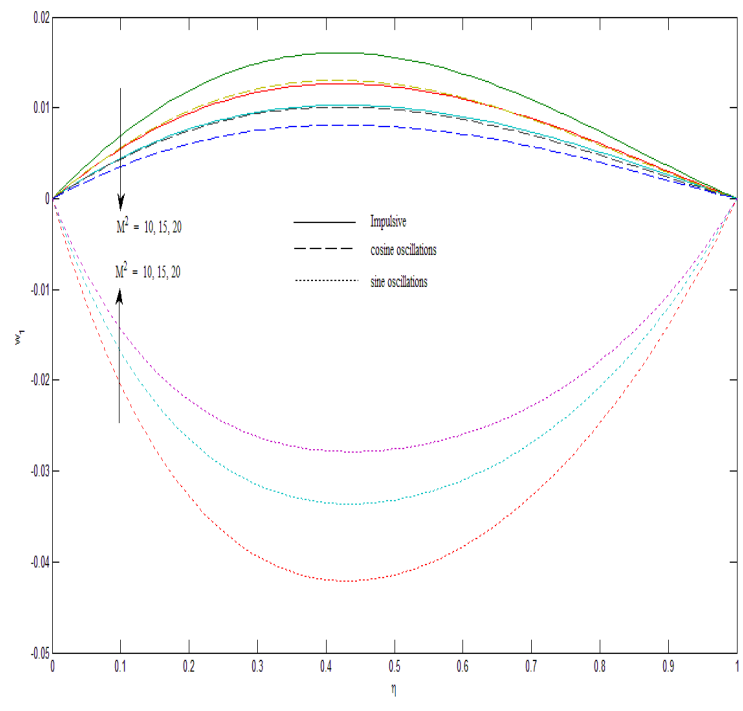

Fig.3: Secondary velocity $w_{1}$ for different $M^{2}$ when $R e=2$, $m=0.2, K^{2}=2, \omega=2$ and $\omega \tau=\frac{\pi}{4}$ 


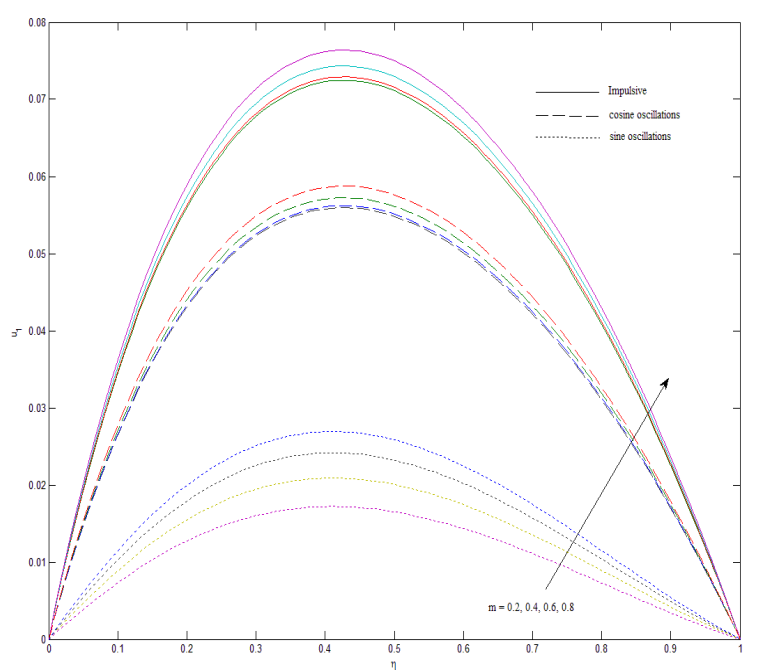

Fig.4: Primary velocity $u_{1}$ for different $m$ when $M^{2}=5$, $R e=2, m=0.2, K^{2}=2, \omega=2$ and $\omega \tau=\frac{\pi}{4}$

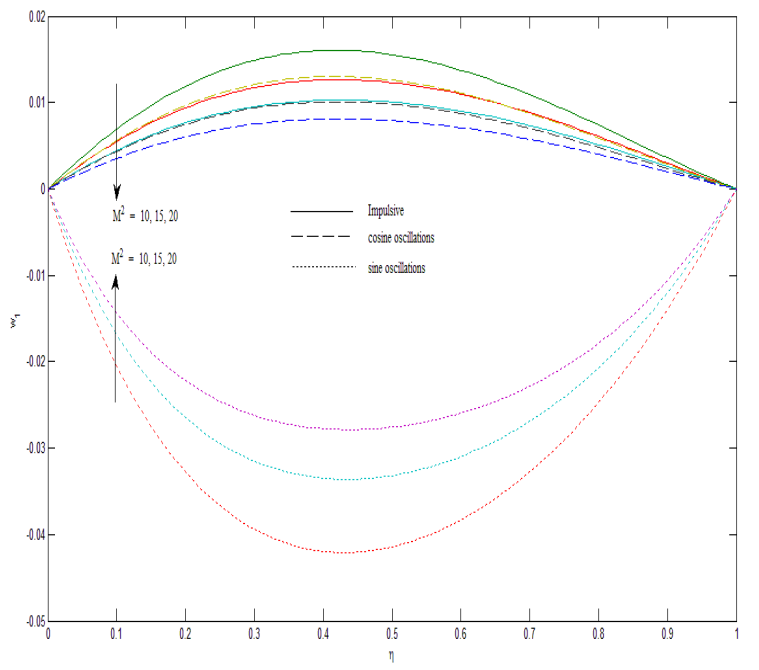

Fig.5: Secondary velocity $w_{1}$ for different $m$ when $M^{2}=5$, $\operatorname{Re}=2, K^{2}=2, \omega=2$ and $\omega \tau=\frac{\pi}{4}$

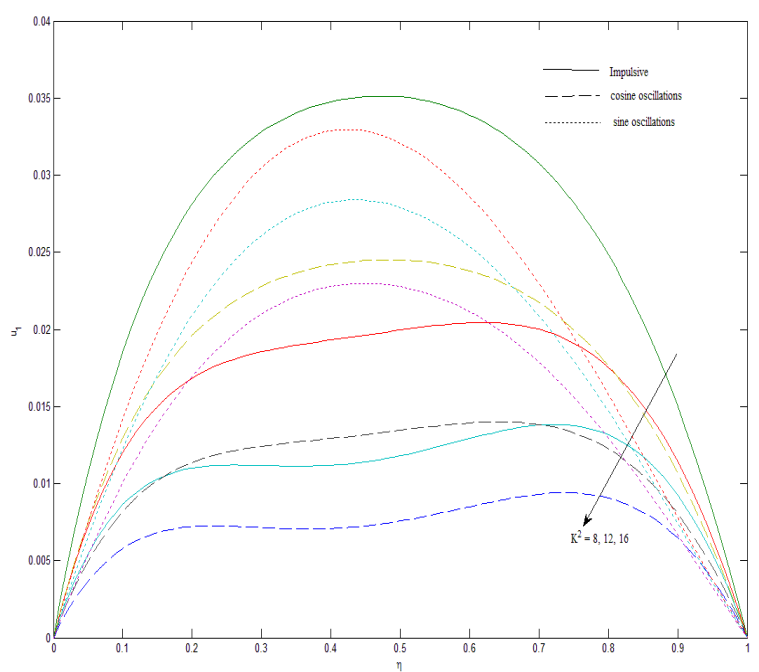

Fig.6: Primary velocity $u_{1}$ for different $K^{2}$ when $M^{2}=5$, $\operatorname{Re}=2, m=0.2, \omega=2$ and $\omega \tau=\frac{\pi}{4}$

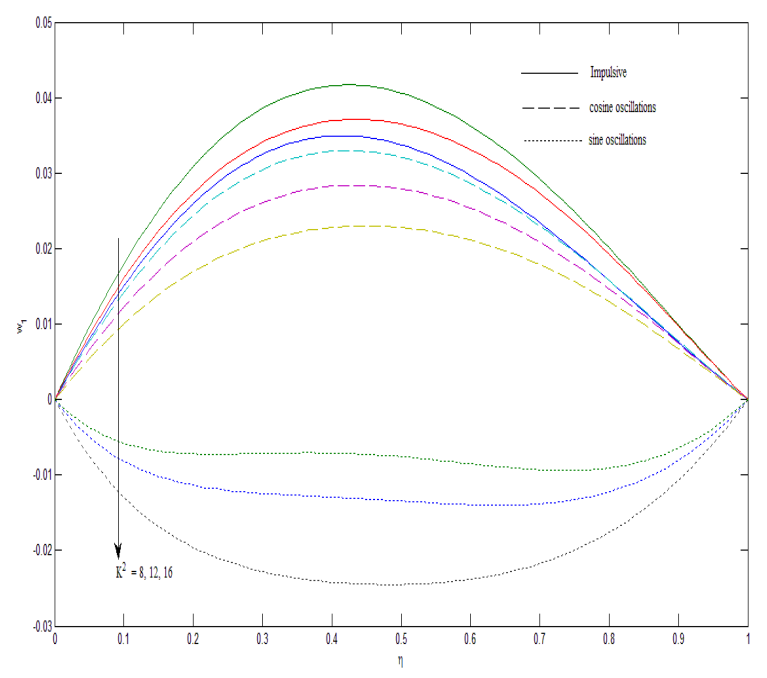

Fig.7: Secondary velocity $w_{1}$ for different $K^{2}$ when $M^{2}=5$, $\operatorname{Re}=2, m=0.2, \omega=2$ and $\omega \tau=\frac{\pi}{4}$ 


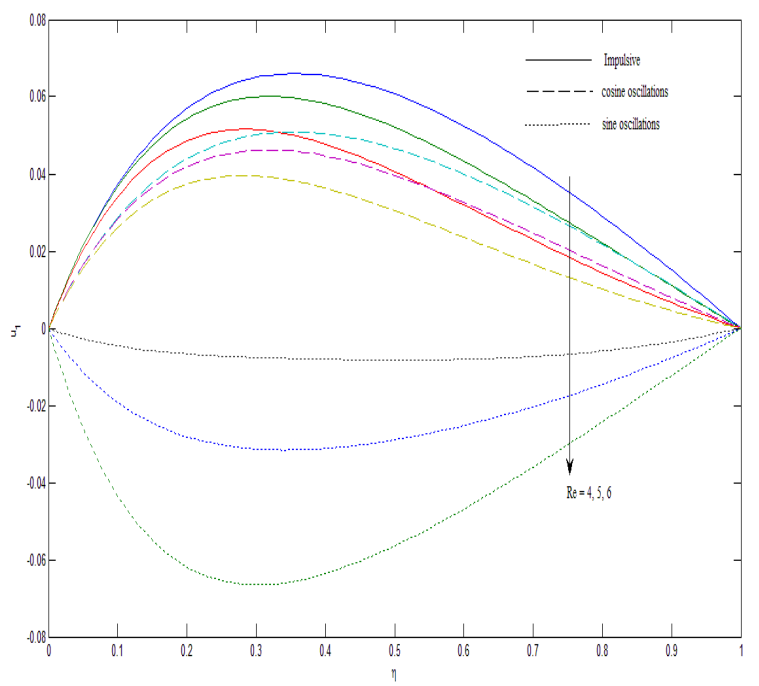

Fig.8: Primary velocity $u_{1}$ for different $\operatorname{Re}$ when $M^{2}=5$, $K^{2}=2, m=0.2, \omega=2$ and $\omega \tau=\frac{\pi}{4}$

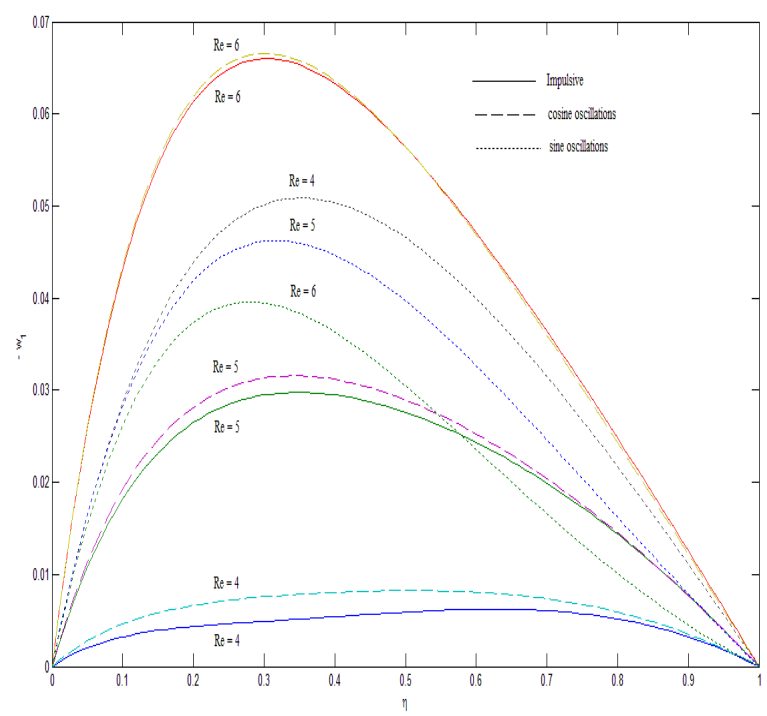

Fig.9: Secondary velocity $w_{1}$ for different $\operatorname{Re}$ when $M^{2}=5$, $K^{2}=2, m=0.2, \omega=2$ and $\omega \tau=\frac{\pi}{4}$

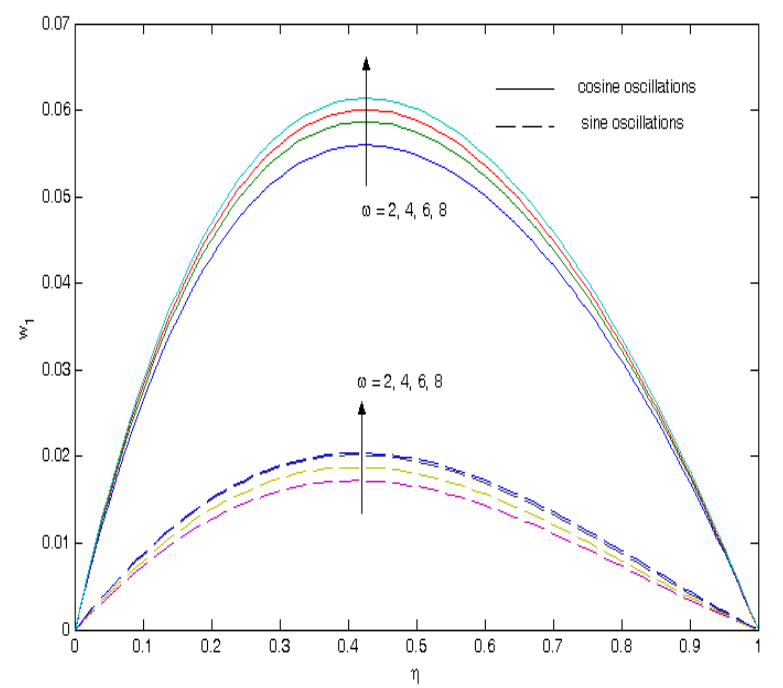

Fig.10: Primary velocity $u_{1}$ for different $\omega$ when $M^{2}=5$, $K^{2}=2, m=0.2, \operatorname{Re}=2$ and $\omega \tau=\frac{\pi}{4}$

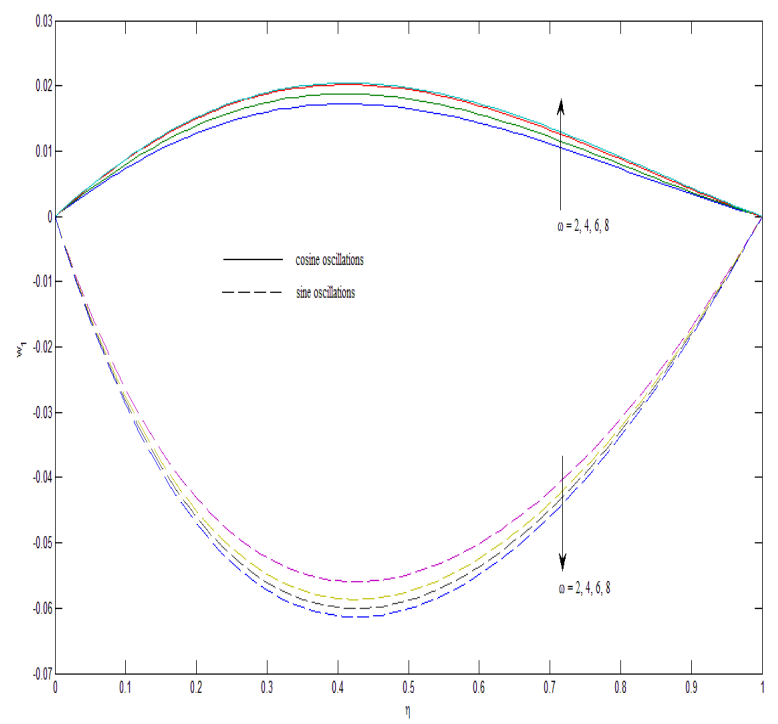

Fig.11: Secondary $w_{1}$ for different $\omega$ when $M^{2}=5, K^{2}=2$, $m=0.2, R e=2$ and $\omega \tau=\frac{\pi}{4}$ 


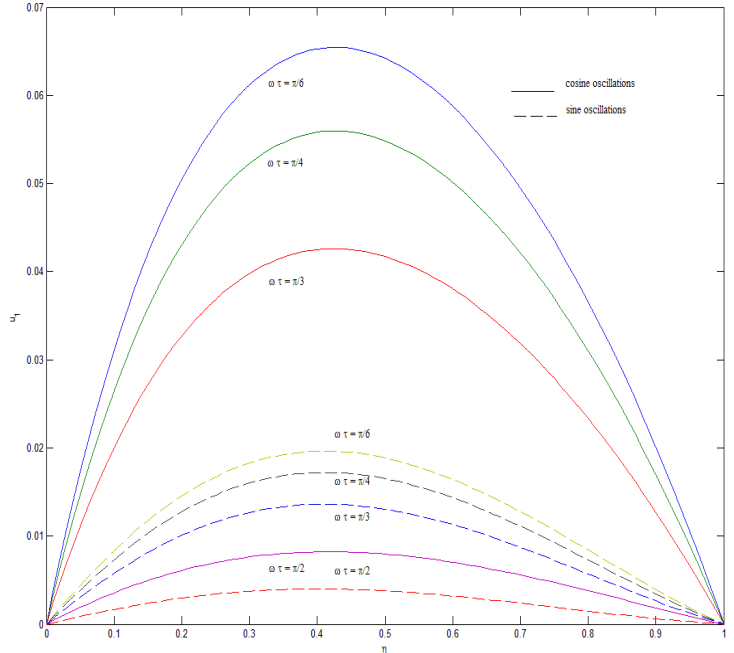

Fig.12: Primary velocity $u_{1}$ for different $\omega \tau$ when $M^{2}=5$, $K^{2}=2, m=0.2, R e=2$ and $\omega=2$

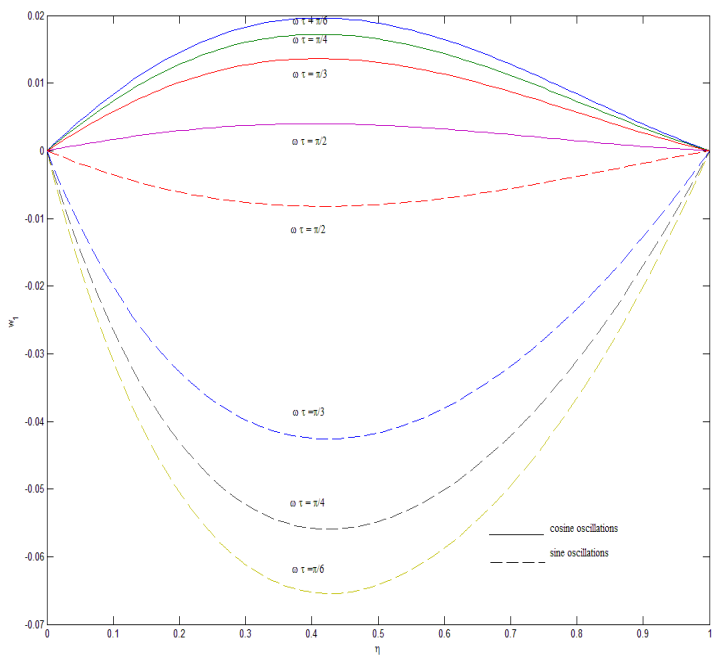

Fig.13: Secondary velocity $w_{1}$ for different $\omega \tau$ when $M^{2}=5$, $K^{2}=2, m=0.2, R e=2$ and $\omega=2$

For the impulsive change of the pressure gradient, the nondimensional shear stresses at the wall $\eta=0$ are given by

$$
\begin{aligned}
\tau_{x}+i \tau_{z}= & \left(\frac{\partial F}{\partial \eta}\right)_{\eta=0} \\
= & \frac{P_{0}}{d}\left[\left\{\frac{1}{2} \operatorname{Re}+(\alpha-i \beta)\right\} \operatorname{coth}(\alpha-i \beta)\right. \\
& \left\{\frac{\left\{\frac{1}{2} \operatorname{Re}-(\alpha-i \beta)\right\}}{\sinh (\alpha-i \beta)} e^{\frac{1}{2} R e}\right]-\frac{P_{0} R e}{d} \operatorname{coth} \frac{R e}{2} e^{-d \tau}
\end{aligned}
$$

$$
+2 P_{0} \pi^{2} \sum_{n=1}^{\infty} \frac{n^{2}}{s_{1}}\left(\frac{e^{s_{1} \tau}}{d+s_{1}}\right)\left[(-1)^{n} e^{\frac{1}{2} R e}-1\right]
$$

where $\alpha, \beta$ and $s_{1}$ are given by (24).

For the cosine oscillations of the pressure gradient, the nondimensional shear stresses at the wall $\eta=0$ are given by

$$
\begin{aligned}
& \tau_{x}+i \tau_{z}=\left(\frac{\partial F}{\partial \eta}\right)_{\eta=0} \\
& =\frac{P}{2}\left[\frac { e ^ { i \omega \tau } } { d + i \omega } \left\{\left(\frac{1}{2} \operatorname{Re}+\left(\alpha_{1} \mp i \beta_{1}\right)\right) \operatorname{coth}\left(\alpha_{1} \mp i \beta_{1}\right)\right.\right. \\
& \left.+\frac{\left(\frac{1}{2} R e-\left(\alpha_{1} \mp i \beta_{1}\right)\right)}{\sinh \left(\alpha_{1} \mp i \beta_{1}\right)} e^{\frac{1}{2} R e}\right\} \\
& +\frac{e^{-i \omega \tau}}{d-i \omega}\left\{\left(\frac{1}{2} \operatorname{Re}+\left(\alpha_{2}-i \beta_{2}\right)\right) \operatorname{coth}\left(\alpha_{2}-i \beta_{2}\right)\right. \\
& \left.\left.+\frac{\left\{\frac{1}{2} \operatorname{Re}-\left(\alpha_{2}-i \beta_{2}\right)\right\}}{\sinh \left(\alpha_{2}-i \beta_{2}\right)} e^{\frac{1}{2} \operatorname{Re}}\right\}\right] \\
& -\frac{P \operatorname{Re}}{2}\left(\frac{1}{d+i \omega}+\frac{1}{d-i \omega}\right) \operatorname{coth} \frac{1}{2} \operatorname{Re}^{-d \tau} \\
& +\pi^{2} P \sum_{n=1}^{\infty} n^{2}\left(\frac{1}{s_{1}-i \omega}+\frac{1}{s_{1}+i \omega}\right)\left(\frac{e^{s_{1} \tau}}{d+s_{1}}\right)\left[(-1)^{n} e^{\frac{1}{2} R e}-1\right],
\end{aligned}
$$

where $\alpha_{1}, \beta_{1}, \alpha_{2}, \beta_{2}$ and $s_{1}$ are given by (24).

For the sine oscillations of the pressure gradient, the nondimensional shear stresses at the wall $\eta=0$ are given by

$$
\begin{aligned}
& \tau_{x}+i \tau_{z}=\left(\frac{\partial F}{\partial \eta}\right)_{\eta=0} \\
& =\frac{P}{2 i}\left[\frac { e ^ { i \omega \tau } } { d + i \omega } \left\{\left(\frac{1}{2} \operatorname{Re}+\left(\alpha_{1} \mp i \beta_{1}\right)\right) \operatorname{coth}\left(\alpha_{1} \mp i \beta_{1}\right)\right.\right. \\
& \left.+\frac{\left\{\frac{1}{2} R e-\left(\alpha_{1} \mp i \beta_{1}\right)\right\}}{\sinh \left(\alpha_{1} \mp i \beta_{1}\right)} e^{\frac{1}{2} R e}\right\} \\
& +\frac{e^{-i \omega \tau}}{d-i \omega}\left\{\left(\frac{1}{2} R e+\left(\alpha_{2}-i \beta_{2}\right)\right) \operatorname{coth}\left(\alpha_{2}-i \beta_{2}\right)\right. \\
& \left.\left.+\frac{\left\{\frac{1}{2} \operatorname{Re}-\left(\alpha_{2}-i \beta_{2}\right)\right\}}{\sinh \left(\alpha_{2}-i \beta_{2}\right)} e^{\frac{1}{2} R e}\right\}\right] \\
& -\frac{P \operatorname{Re}}{2 i}\left(\frac{1}{d+i \omega}+\frac{1}{d-i \omega}\right) \operatorname{coth} \frac{1}{2} \operatorname{Re}^{-d \tau} \\
& -\pi^{2} P i \sum_{n=1}^{\infty} n^{2}\left(\frac{1}{s_{1}-i \omega}+\frac{1}{s_{1}+i \omega}\right)\left(\frac{e^{s_{1} \tau}}{d+s_{1}}\right)\left[(-1)^{n} e^{\frac{1}{2} R e}-1\right],
\end{aligned}
$$


where $\alpha_{1}, \beta_{1}, \alpha_{2}, \beta_{2}$ and $s_{1}$ are given by (24).

Numerical results of the non-dimensional shear stresses $\tau_{x}$ and $\tau_{z}$ at the wall $\eta=0$ due to the primary and the secondary flows are presented in Figs.14-23 against magnetic parameter $M^{2}$ for several values of Hall parameter $m$, rotation parameter $K^{2}$, Reynolds number Re, frequency parameter $\omega$ and phase angle $\omega \tau$. Fig.14 shows that the shear stress $\tau_{x}$ due to the primary flow at the wall $\eta=0$ decreases for both impulsive change and cosine oscillations of the pressure gradient while it increases for sine oscillations of the pressure gradient with an increase in Hall parameter $m$. It is seen from Fig. 15 that the shear stress $\tau_{z}$ due to the secondary flow at the wall $\eta=0$ increases for the impulsive change, cosine and sine oscillations of the pressure gradient with an increase in Hall parameter $m$. Fig.16 shows that the shear stress $\tau_{x}$ due to the primary flow increases when $M^{2}<2.8$ and then it decreases for the impulsive change and cosine oscillations while it increases for sine oscillations of the pressure gradient with an increase in rotation parameter $K^{2}$. It is found from Fig.17 that the shear stress $\tau_{z}$ decreases for both impulsive change and cosine oscillations of the pressure gradient while it increases for sine oscillations of the pressure gradient with an increase in rotation parameter $K^{2}$. Fig. 18 shows that the shear stress $\tau_{x}$ decreases for small values of magnetic parameter $M^{2}$ and then it increases for the impulsive change, cosine and sine oscillations of the pressure gradient with an increase in Reynolds number Re. It is seen from Fig.19 that the shear stress $\tau_{z}$ increases for both impulsive change and cosine oscillations of the pressure gradient while it decreases for sine oscillations of the pressure gradient with an increase in Reynolds number Re. Fig.20 shows that the shear stress $\tau_{x}$ increases for small values of magnetic parameter $M^{2}$ and then it decreases for cosine and sine oscillations of the pressure gradient with an increase in frequency parameter $\omega$. It is found from Fig.21 that the shear stress $\tau_{z}$

increases for small values of magnetic parameter $M^{2}$ and then it decreases for cosine oscillations while it first decreases and then increases for sine oscillations of the pressure gradient with an increase in frequency parameter $\omega$. Fig.22 shows that the shear stress $\tau_{x}$ decreases for both cosine and sine oscillations of the pressure gradient with an increase in phase angle $\omega \tau$. It is seen from Fig.23 that the shear stress $\tau_{z}$ decreases for cosine oscillations of the pressure gradient while it increases for sine oscillations of the pressure gradient with an increase in phase angle $\omega \tau$.

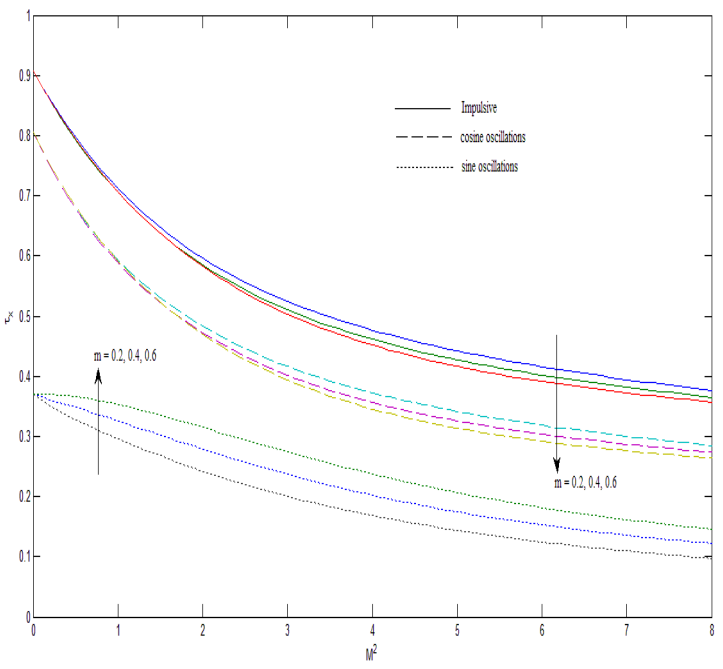

Fig.14: Shear stress $\tau_{x}$ for different $m$ when $\omega=2, K^{2}=2$, $R e=2$ and $\omega \tau=\frac{\pi}{4}$

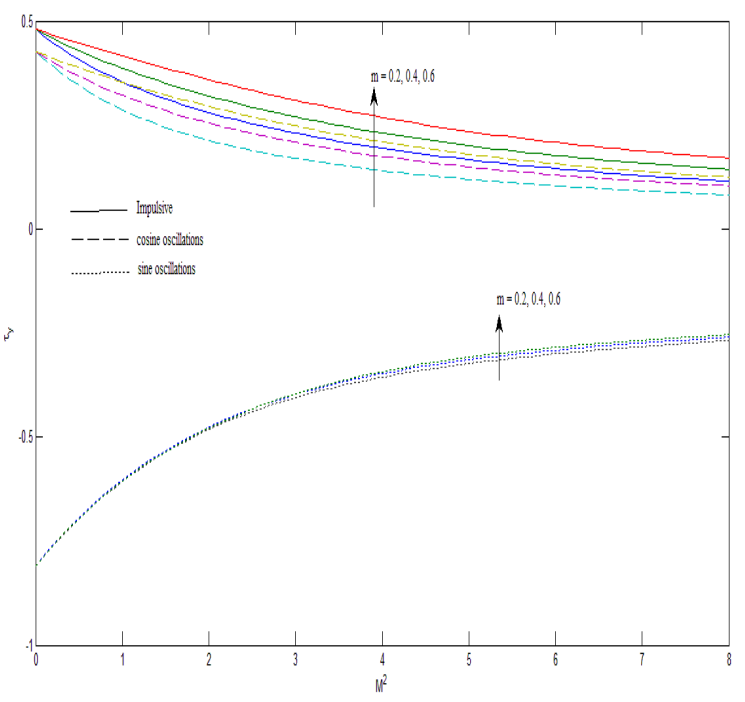

Fig.15: Shear stress $\tau_{z}$ for different $m$ when $\omega=2, K^{2}=2$, $R e=2$ and $\omega \tau=\frac{\pi}{4}$ 


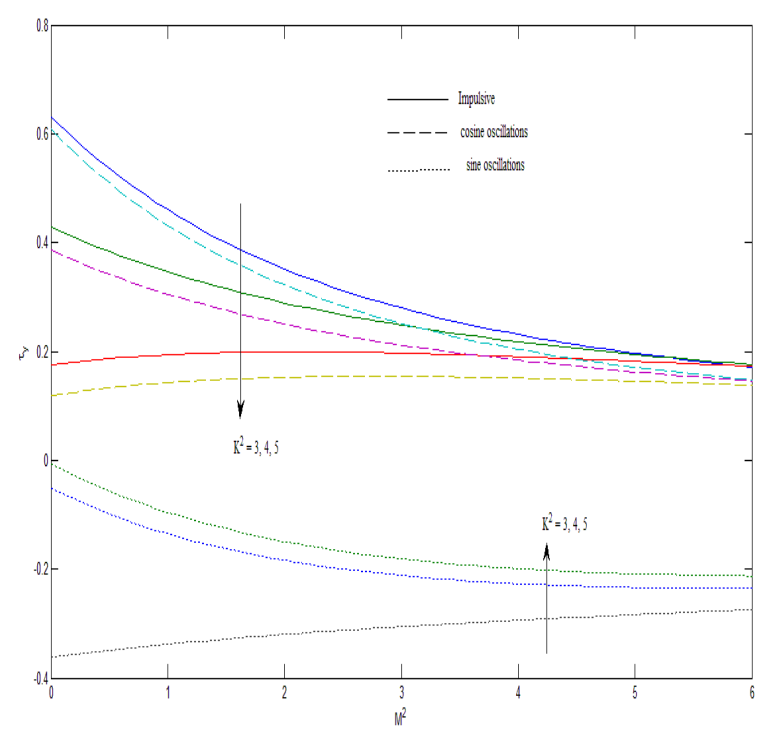

Fig.16: Shear stress ${ }^{\tau_{x}}$ for different $K^{2}$ when $\omega=2, m=0.2$ , $R e=2$ and $\omega \tau=\frac{\pi}{4}$

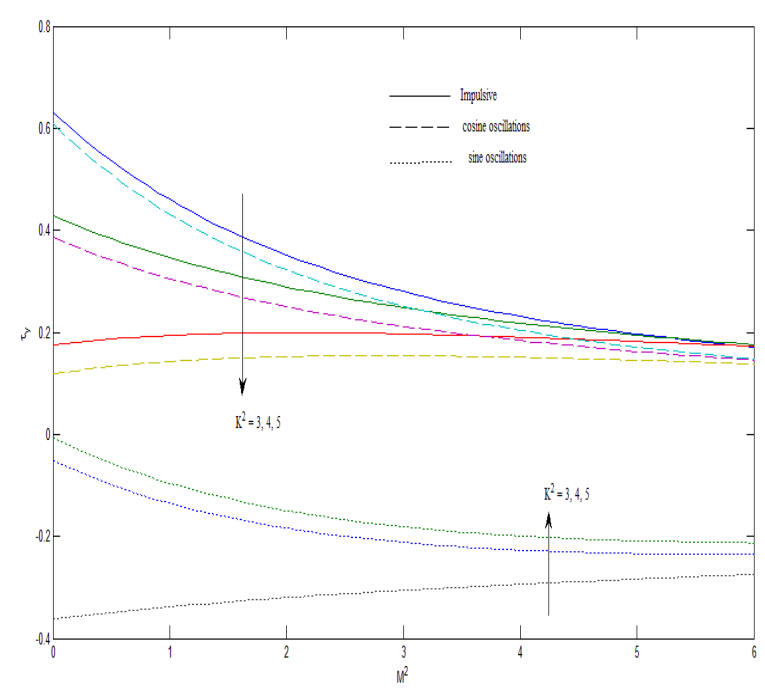

Fig.17: Shear stress ${ }^{\tau_{z}}$ for different $K^{2}$ when $\omega=2, m=0.2$ , $R e=2$ and $\omega \tau=\frac{\pi}{4}$

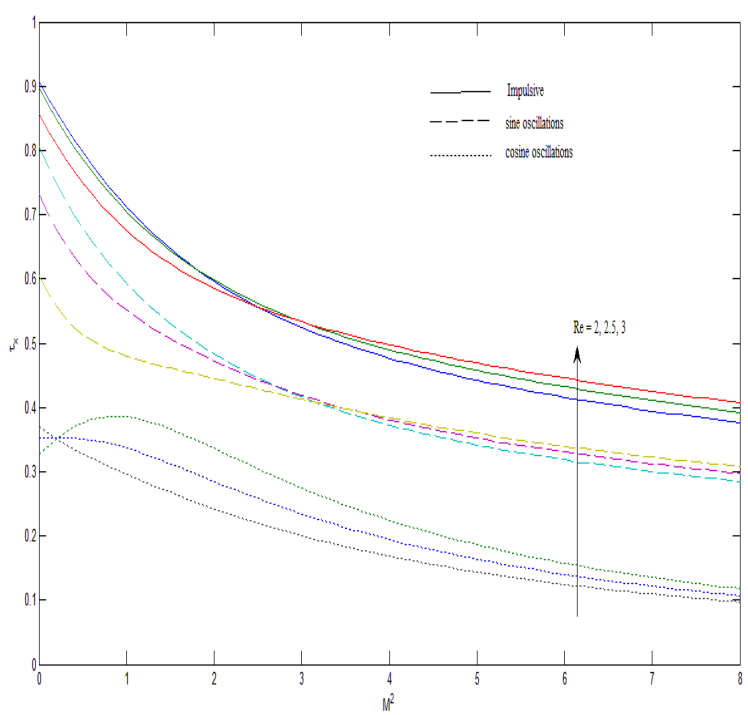

Fig.18: Shear stress $\tau_{x}$ for different $\operatorname{Re}$ when $\omega=2, m=0.2$ $K^{2}=2$ and $\omega \tau=\frac{\pi}{4}$

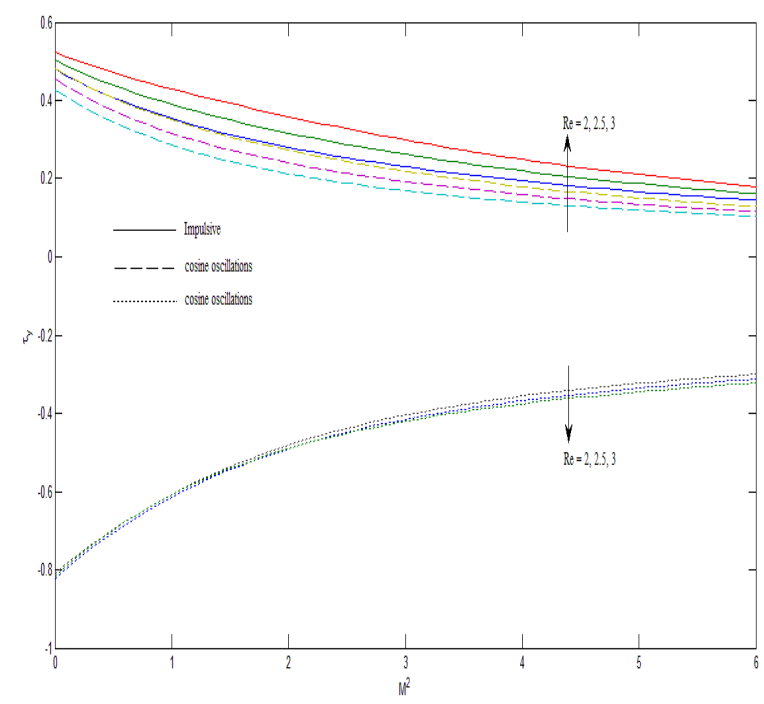

Fig.19: Shear stress ${ }^{\tau_{z}}$ for different $\operatorname{Re}$ when $\omega=2, m=0.2$ $K^{2}=2$ and $\omega \tau=\frac{\pi}{4}$ 


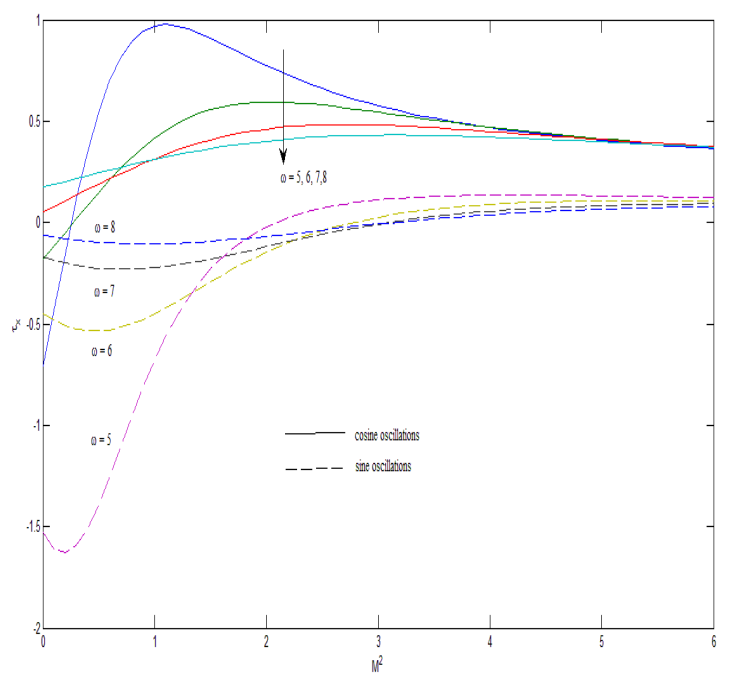

Fig.20: Shear stress $\tau_{x}$ for different $\omega^{\omega}$ when $R e=2, m=0.2$, $K^{2}=2$ and $\omega \tau=\frac{\pi}{4}$

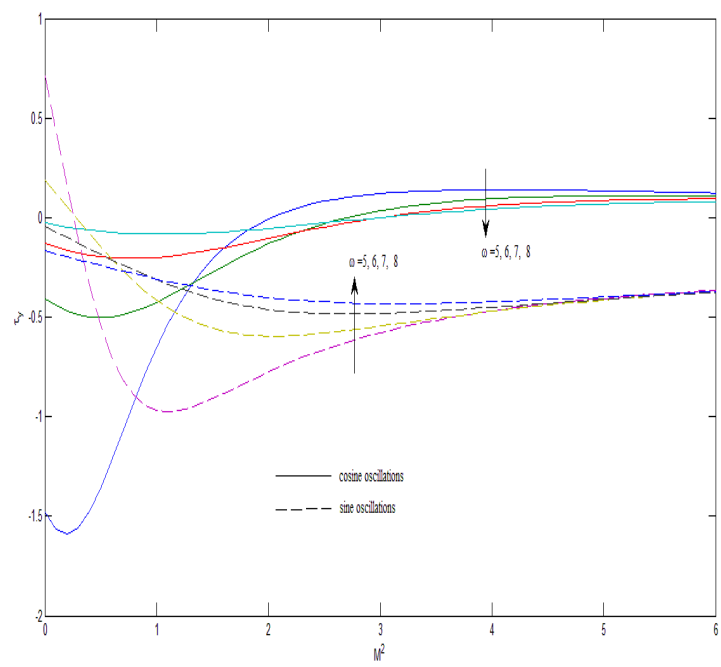

Fig.21: Shear stress ${ }^{\tau_{z}}$ for different $\omega$ when $R e=2, m=0.2$, $K^{2}=2$ and $\omega \tau=\frac{\pi}{4}$

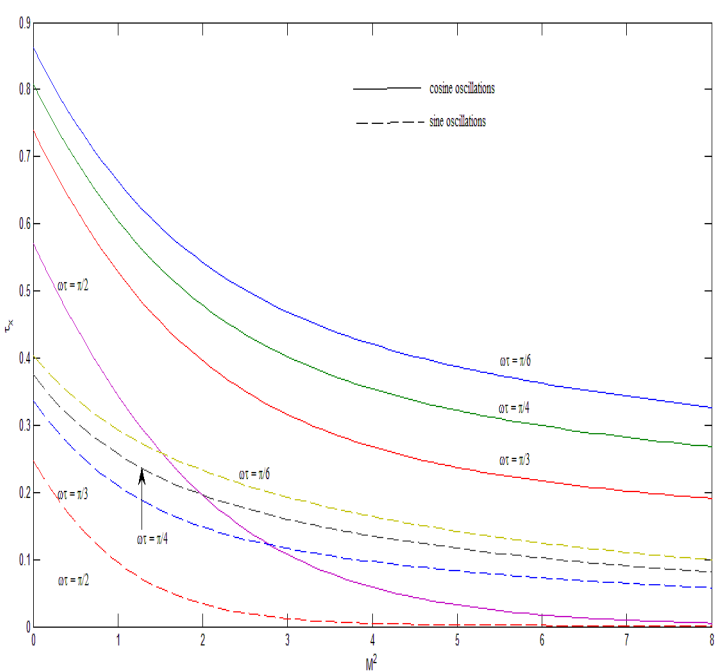

Fig.22: Shear stress $\tau_{x}$ for different $\omega \tau$ when $R e=2$, $m=0.2, K^{2}=2$ and $\omega=2$

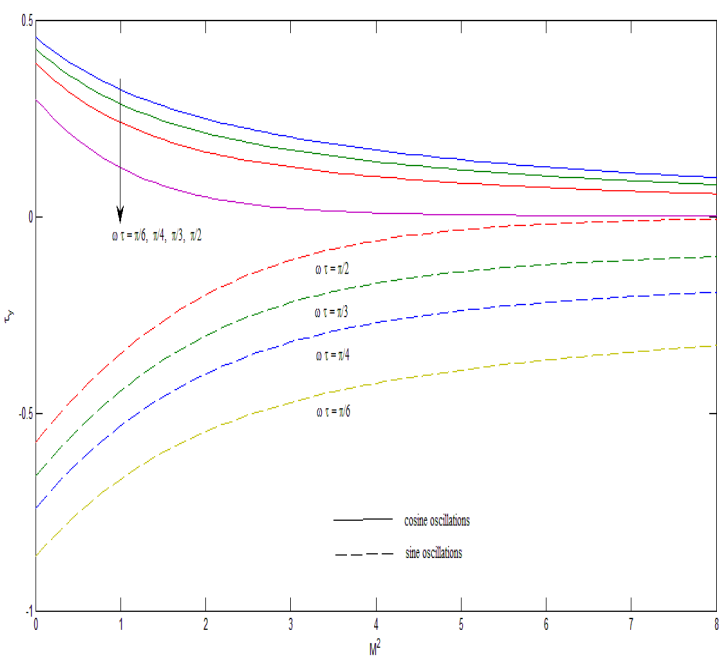

Fig.23: Shear stress $\tau_{z}$ for different $\omega \tau$ when $R e=2, m=0.2$, $K^{2}=2$ and $\omega=2$

\section{CONCLUSION}

Hall effects on the unsteady MHD flow in a horizontal channel with variable pressure gradient in a rotating system have been investigated. It is found that the primary velocity increases with an increase in Hall parameter for the impulsive change, cosine and sine oscillations of the pressure gradient. The secondary velocity increases for the impulsive change and cosine oscillations while it decreases for sine oscillations of the pressure gradient with an increase in Hall parameter. The primary and secondary velocities decrease with an increase in rotation parameter for the impulsive change, cosine and sine oscillations of pressure gradient. Further, the shear stress due to the primary flow at the lower wall reduces for both the impulsive change and cosine oscillations of the pressure pressure whereas it increases for sine oscillations of the pressure gradient with an increase in Hall parameter. The Hall 
parameter increases the shear stress due to the secondary flow at the lower wall for the impulsive change, cosine and sine oscillations of the pressure gradient.

\section{REFERENCES}

[1] Hide, R. and Roberts, P.H. (1961). The origin of the mean geomagnetic field, in: Physics and Chemistry of the Earth, vol. 4, Pergamon Press, New York, pp. 27-98.

[2] Dieke, R.H. (1970). Internal rotation of the sun, in: L. Goldberg (Ed.), Annual Reviews of Astronomy and Astrophysics, vol. 8, Annual Reviews Inc, pp. 297-328.

[3] Sutton, G.W. and Sherman, A.(1965). Engineering Magnetohydrodynamics. McGraw-Hill, New York.

[4] Sato, H.(1961). The Hall effects in the viscous flow of ionized gas between parallel plates under transverse magnetic field, J. Phys. Soc. Japn., 16: 14-27.

[5] Nanda, R.S., Mohanty, H.K. (1970). Hydromagnetic rotating channel flows, Appl. Sci. Res., A 24: 65-75.

[6] Datta, N. and Jana, R. N.(1977). Hall effects on unsteady Couette flow, Int. J. Engng. Sci., 15: 35-43.

[7] Datta, N. and Jana, R. N.(1977). Hall effects on hydromagnetic convective flow through a channel with conducting walls, Int. J. Eng. Sci., 15: 561.

[8] Mandal, G., Mandal, K. K. and Choudhury, G. (1982). On combined effects of Coriolis force and Hall current on steady MHD Couette flow and heat transfer, J. Phys. Soc. Japan, 51: 2010-2015.

[9] Mandal, G. and Mandal, K. K.(1983). Effects of Hall current on MHD Couette flow between thick arbitrarily conducting plates in a rotating system, J. Phys. Soc. Japan, 52:470.

[10] Ghosh SK (1993). Unsteady hydromagnetic flow in a rotating channel with oscillating pressure gradient, J. Phys. Soc. Jpn, 62: 3893-3903.

[11] Nagy, T. and Demandy, Z.(1995). Effects of Hall currents and Coriolis force on Hartmann flow under general wall conditions, Acta Mechanica, 113: 77-91.

[12] Kanch, A. K. and Jana, R. N.(2001). Hall effect on unsteady Couette flow under boundary layer approximations, $J$. Physical Sciences, 7: 74-86.

[13] Ghosh, S.K.(2002). Effects of Hall current on MHD Couette flow in a rotating system with arbitrary magnetic field, Czech. J. Phys., 52: 51-63.

[14] Ghosh, S. K. and Pop, I.(2004). Hall effects on MHD plasma Couette flow in a rotating environment, Int. J. Applied Mech. Eng., 9(2): 293-305.

[15] Ghosh, S. K.(2002): Effects of Hall current on MHD Couette flow in a rotating system with arbitrary magnetic field, Czech. J. Phys., 52(1): 51-63.

[16] Guria, M. and Jana, R. N.(2007). Hall effects on the hydromagnetic convective flow through a rotating channel under general wall conditions, Magnetohydrodynamics, 43(3): 287-300.

[17] Attia, H.A. (2009). Ion Slip effects on unsteady Couette flow with heat transfer under exponential decaying pressure gradient, Tamkang Journal and Engineering, 12(2): 209214.

[18] Seth, G. S., Nandkeolyar, R. and Ansari, Md. S.(2009). Hall effects on oscillatory hydromagnetic Couette flow in a rotating system, Int. J. Acad. Res.1: 6.
[19] Chauhan, D.S. and Rastogi, P. (2009). Hall current and heat transfer effects on MHD flow in a channel partially filled with a porous medium in a rotating system, Turkish J. Eng. Env. Sci., 33:167-184.

[20] Chauhan, D.S. and Agrawal, R.(2010). Effects of Hall current on MHD flow in a rotating channel partially filled with a porous medium, Chemical Engineering Communications, 197(6):830-845.

[21] Jha, B. K. and Apere, C. A. (2010). Combined effects of Hall current and ion-slip current on unsteady MHD Couette flow in a rotating system, J. Physical Society of Japan, 79(10): 104401(1-9)

[22] Guchhait, S. K., Das, S., Jana, R. N. and Ghosh, S. K.(2011). Combined effects of Hall current and rotation on unsteady Couette flow in a porous channel, World Journal of Mechanics, 1: 87-99.

[23] Das, S., Sarkar, B. C. and Jana, R. N.(2011). Hall effects on MHD Couette flow in rotating system, Int. J. Com. Appl., 35(13): 22-30

[24] Ghara, N., Maji, S. L., Das, S., Jana, R. N. and Ghosh, S.K.(2012). Effects of Hall current and ion-slip on unsteady MHD Couette flow, Open Journal of Fluid Dynamics, 2: 113.

[25] Seth, G. S., Nandkeolyar, R. and Ansari, Md. S.(2012): Effects of Hall current and rotation on unteady MHD Couette flow in the presence of an inclined magnetic field, J. Applied Fluid Mechanics, 5(2): 67-74.

[26] Chauhan, D.S. and Agrawal, R. (2012). Effects of hall current on MHD Couette flow in a channel partially filled with a porous medium in a rotating system, Meccanica, 47: 405-421.

[27] Sarkar, B. C., Das, S. and Jana, R. N. (2013). Combined effects of Hall currents and rotation on steady hydromagnetic Couette flow, Research Journal of Applied Sciences, Engineering and Technology, 5(6): 1864-1875.

[28] Nadeem, S., Akbar, N. S. and Malik, M. Y. (2010). Numerical solutions of peristaltic flow of a newtonian fluid under the effects ofmagnetic field and heat transfer in a porous concentric tubes, Zeitschrift fur Naturforschung, 65a(5): 369-380.

[29] Nadeem, S. and Akbar, N.S. (2011). Influence of heat transfer and variable viscosity in vertical porous annulus with peristalsis, Journal of Porous Media, 14: 849-863.

[30] Nadeem, S., Akbar, N. S., Hayat, T. and Hendi, A. A.(2012). Influence of heat and mass transfer on Newtonian biomagnetic fluid of blood flow through a tapered porous arteries with a stenosis, Transport in Porous Media, 91: 81100.

[31] Akbar, N. S. and Nadeem, S.(2012). Simulation of variable viscosity and Jeffrey fluid model for blood flow through a tapered artery with a stenosis, Communications in Theoretical Physics, 57: 133-140.

[32] Akbar, N. S. and Nadeem, S.(2012). Analytical and numerical analysis of Vogel's model of viscosity on the peristaltic flow of Jeffrey fluid, Journal of Aerospace Engineering, 25: 64-71.

[33] Cowling, T. G., Magnetohydrodynamics, New York, Intersscience, 1957. 